\title{
Using Bacterial Vectors for Probable Vaccines: From Molecular Mechanism to Cancer Therapy
}

\author{
Parnia Jabbari \\ Department of New Medical Science, Islamic Azad University Tehran Medical Branch, Tehran, Iran \\ Email: parniajabbari9@gmail.com
}

How to cite this paper: Jabbari, P. (2020) Using Bacterial Vectors for Probable Vaccines: From Molecular Mechanism to Cancer Therapy. World Journal of Vaccines, 10, 33-42.

https://doi.org/10.4236/wjv.2020.102003

Received: February 29, 2020

Accepted: May 27, 2020

Published: May 30, 2020

Copyright $\odot 2020$ by author(s) and Scientific Research Publishing Inc. This work is licensed under the Creative Commons Attribution International License (CC BY 4.0).

http://creativecommons.org/licenses/by/4.0/

\begin{abstract}
Anti-cancer therapies over the few decades, faced with many challenges. And bacterial vaccine vectors have shown a potential to be replaced as the cutting-edge technology for such aspects. Bacterial vaccine vectors with a suitable DNA can be a potential option for cancer treatment as a carrier for tumoricidal agents or bacterially directed Enzyme Prodrug treatment. Throughout this study, it is planned to have a review of the use of bacteria as vehicles by different ways for cancer treatment, detailing the systems of function and achievements at preclinical and clinical levels.
\end{abstract}

\section{Keywords}

Bacterial Vector, Vaccine Delivery System, Cancer Vaccine, DNA Vaccine, Tumor

\section{Introduction}

Cancer is one of the series causes of death in developing countries [1] [2]. Solid tumors are identified by the hypoxia and necrosis regions, leading to high metabolic activity of the tumor tissues and lack of oxygen supply due to inadequate vasculature [3] [4]. The hypoxic areas of solid tumors possess increased resistance to conventional therapies for cancer ranging from radiotherapy to chemotherapy in compression to better-oxygenated tumor tissues. And due to a wide array of factors that bring about development of drug-resistant tumor cells presence of undetectable micro-metastases at the time of diagnosis and treatment, this has led to the advent of new approaches to cancer therapy, for instance, the delivery of anti-cancer genes to the tumor spots by means of bacteria [5] [6].

Bateria are being used for the sake of vaccinating the host or as oncolytic vec- 
tors that not only can directly kill tumor cells, but also stimulate anti-tumor immune responses [7] [8]. Especially in patients with highly advanced cancer who are resistant to conventional treatments, these treatments can produce tumor regression with minimal toxicity [9] [10] (Figure 1). The first bacteria observed to have a far-reaching effect on cancer cells appertain to the Clostridium genus. In 1813, tumors regression was observed in patients who developed concomitant "gas gangrene" brought by $C$. perfringens [11]. Those provide specific features such as: gram-positive, spore-forming, obligate anaerobes and many strains are pathogenic. It has previously been established for many species of Clostridium injecting in spore form, germinating only once in an appropriate anaerobic environment. Some Bacteria have a potential to be used in vectors like: Bacille Calmette-Guerin (BCG), Listeria monocytogenes, Salmonella species, Vibrio cholera, Bifidobacterium [12] [13] [14], Listeria monocytogenes [15] [16], Bordetella pertussis [17], Shigella spp [17] [18], and Escherichia coli [19] [20].

Bacterial vaccine vectors trigger to a wide range of privileges: 1) there are several mutations that are weakened with viral secretion; 2 ) the quantity and in vivo location of antigen expression can be regulated; 3) different pathways of the vaccine are possible; and 4) they have a potential to adapt themselves with innate immune system stimulators. These bacterial vaccine vectors can be used to supply protection against self-antigens as well as heterologous antigens. For example, attenuated Salmonella typhimurium vectors have been used to produce protective immune responses in mice and in some cases humans against cancer. The diversity and immunogenicity of this platform make it a great vector for vaccines [21], although the safety of these vectors that are live genetically transformed organisms should not be ignored for both patients and the environment. Thus, the optimal bacteria type (species and mode of antigen delivery to APCs), the strategy to reduce the severity of the disease, dissemination, and route of vaccine administration remains to be determined [22]. Also, regarding chief problems of pathogenic bacteria as vaccine vectors should be said that all cancer vaccines need to overcome peripheral $\mathrm{T}$ cell tolerance against tumor self-antigens that as a barrier have an influence on effective anti-tumor immune responses [23]. The bacterial-vectored vaccines have an induction of systemic T-cell responses, including polyfunctional cytokine-secreting CD41 and CD81 T-cells [24].

Another thing worth mentioning is pathogenic re-version as a result of recombination events or horizontal gene transfer that is practically eliminated, and others are complications that can arise due to pre-existing immunity. Prior disposal to the bacterial vector has been demonstrated to diminish the efficacy of the vaccine [25]. But still, over the last 200 years [26] [27], combination of DNA with bacteria serves as vectors to deliver anti-cancer agents, cytotoxic peptides, therapeutic proteins or prodrug converting enzymes to solid tumors. On the other hand, some type of them for protection and mediation for tissue or cell are being used [13] [28]. Here, we describe the advances made in these areas to initiate a new era of live bacterial vaccines. 


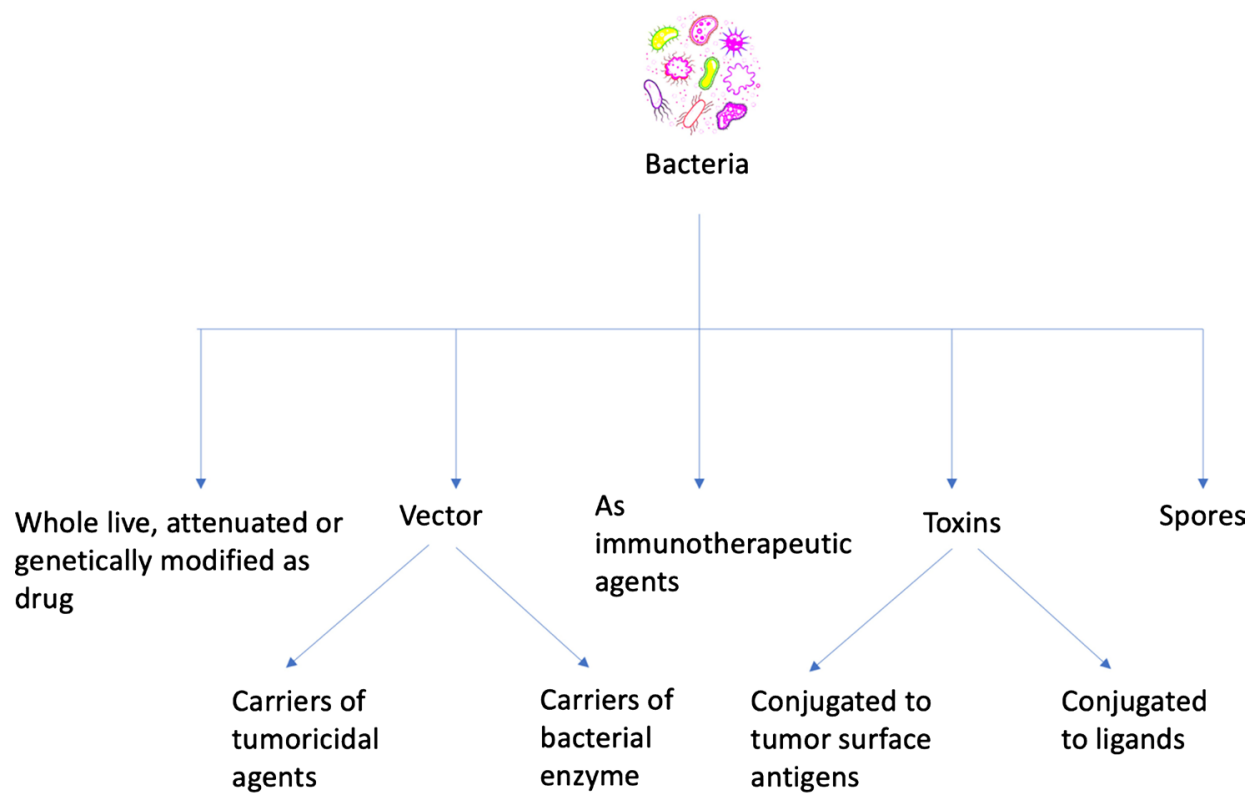

Figure 1. All approaches that bacteria might be used in cancer treatment.

\section{Bacteria as the Vector for Difference Preference; Carrier of Tumoricidal Factors, the Carrier of the Bacterial Enzyme}

\subsection{Bacteria as Carriers of Tumoricidal Factors}

An attenuated form of $S$. typhimurium has the utmost propriety to deliver cytokines locally to liver, with an effect on hepatic metastases as it naturally colonizes in liver. A genetically engineered strain of $S$. typhimurium has been developed which is cya/crp mutant (genes encoding proteins involved in the regulation of cyclic AMP) and expresses interleukin-2 for the treatment of liver cancer in preclinical models [29] [30]. Eukaryotic presentation vectors containing human interleukin-12 (hIL-12), human granulocyte/macrophage colony-stimulating factor (hGM-CSF), mouse (m)IL-12, mGM-CSF and green fluorescent protein (GFP) were used to transform attenuated Salmonella (SL3261). It has been represented that oral administration of Salmonella by the expression of mGM-CSF or mGM-CSF with MIL-12 induces tumor regression in mice bearing lewis lung carcinomas. Tumor cells those faced with genetic modification generate active-protein gene of GM-CSF in the local environment of tumor cells, in particular, activating the patient's $\mathrm{T}$ cells to diminish tumors in metastatic sites [14] [31].

Bifidobacterium adolescentis has been in consideration as a delivery system for the anti-angiogenic protein endostatin [32]. There are several benefits of selecting them as a gene delivery vector for cancer gene therapy: 1) Bifidobacterium is an anaerobic bacterium in the human body that does not produce endotoxin and toxin [33]; 2) Bifidobacterium enhances immune response and inhibits many tumor growths in vivo such as liver cancer, breast cancer, and the like [34];3) 
Bifidobacterium could be easily killed in antibiotics or in an in vivo oxygen environment and it also was confirmed that both wild-type and genetically engineered $B$. adolescentis were killed easily with kanamycin, cefoper-azone, and penicillin in vitro; 4) when B.adolescentis was injected intravenously, bacilli only germinated and proliferated in solid tumor but not found in other normal tissues; 5 ) it demonstrated that $B$. adolescentis has an endostatin gene, which is located in a solid tumor, and selectively inhibits the angiogenesis and growth of hypoxic tumors. These outcomes show $B$. adolescentis could be used as a highly specific gene delivery vector [32].

\subsection{Bacterially Directed Enzyme Prodrug Therapy}

Another remarkable way to overcome the unacceptable side effects of bacterial therapy is the tumor-selective activation of prodrugs. For this method, anaerobic bacteria have been transmitted with an enzyme that can convert a non-toxic prodrug into a toxic drug. As the bacteria develop in the necrotic and hypoxic areas of the tumor, the enzyme is expressed selectively in the tumor. And from the management of prodrug can be understood that prodrug gets metabolized to the toxic drug only in the tumor [35].

For more efficacy, both the prodrug and the activated drug should be able to move cross biological membranes, because the prodrug will be activated in the bacterial cells and then the active drug is introduced into the tumor cells. Several enzyme/prodrug systems are available. Sporgenes is a stain of clostridial has the highest levels of tumor colonization with the E.coli cytosine deaminase (CD) gene and illustrate that systemically injected spores of these bacteria express CD only in the tumor. This enzyme can convert the nontoxic prodrug 5-fluorocytosine (5-FC) to the anticancer drug 5-fluorouracil (5-FU). Furthermore, systemic delivery of 5-FC in mice previously injected with CD-transformed spores of C.sporogenes produced a greater antitumor effect than maximum 5-FU. CD expressed in Clostridium acetobutylicum has shown the selective delivery of an external enzyme to tumors. As far as most human solid tumors possess hypoxic and necrotic areas this kind of vector system is helpful for tumor-selective gene therapy [36] [37].

Moreover, following administration of the vascular targeting agent combretastatin A-4 phosphate significantly increased levels of cytosine deaminase were detected at the tumor site as a consequence of expanded tumor necrosis and subsequently improved growth of $C$. acetobutylicum. The conspicuous results illuminated the potential use of Clostridium-based therapeutic protein transfer to tumors [37].

Salmonella vector combined with NR and CD has shown successful results in vivo and both are currently sustaining phase I clinical trials in cancer patients. Salmonella has been combined with carboxypeptidase G2 (CPG2), an enzyme that binds a wide range of mustard protein to DNA binding agents and exhibits high levels of activity in vivo. TAPET (Tumour Amplified Protein Expression 
Therapy) uses $V N P 20009$, an attenuated strain of $S$. typhimurium as a bacterial vector and displays an $E$. coli $\mathrm{CD}$ for specifically delivering anticancer drugs to solid tumors [38]. The expression of the prodrug, which converts the HS- thymidine kinase (TK) enzyme into a purine action type, soars the anti-tumor activity upon the addition of ganciclovir, the corresponding prodrug [39].

Genus $B$ longum of Bifidobacterium longum is used to achieve delivery of specific tumor, and enzyme-prodrug therapy [40]. B-glucuronidase and the luxCDABE gene cluster were expressed in the DH5a strain of E. coli to generate DH5a-lux/bG. These bacteria gave out light for imaging and hydrolyzed the glucuronide prodrug 9ACG to the topoisomerase that inhibited 9-aminocamptothecin (9AC) colony- forming units (CFUs) and staining for bG activity have indicated that this bacterial strain localizes and replicates in human tumor xenografts thereby generating antitumor activity along with systemic 9ACG prodrug therapy [41]. That reveals bacteria as prodrug's activator have a high potential for cancer chemotherapy.

\section{Cancer Vaccine Targets}

The validation of a target for a therapeutic cancer vaccine directly relates to the ability of a tumor cell to process the tumor-associated antigen (TAA) represented by the vaccine in the context of a peptide-major histocompatibility complex (MHC) for T-cell detection or on the surface of the tumor cell for B-cell recognition. The level of TAA expression in the tumor, the relative property of the TAA for tumor versus normal adult tissue, and the degree of tolerance inherent to the TAA are also of importance [42] [43] [44].

Owing to the antigen is presented in a small amount, immunogenicity is relying upon help from CpG island hypermethylation, which is located in the plasmid spine sequence, playing a vital role as adjuvants, and increasing the induction of $\mathrm{T}$ cell response [45] [46]. The core CpG motif consists of an unmethylated $\mathrm{CpG}$ flanked by two 3 ' pyrimidines and two 5 ' purines. Compared with other bacterial genomes, $\mathrm{CpG}$ sites are not common. Toll-like receptors (TLRs) 9 are expressed in endosomal APCs, including B cells and dendritic cells (DCs) [47]. Varied vaccine platforms have now been measured in Phase II and/or Phase III clinical trials such as; bacteria, whole tumor cells, or delivery of protein-peptide-activated dendritic cells (DCs) [14] [48].

Peptide vaccines have been added to cytokines such as GM-CSF, which increase the activity of DNA and antigen presentation. Peptide vaccines can also be produced from autologous or allogeneic tumor cells. Patients those receiving peptide vaccines should have the same HLA Haplotype as the synthetic peptide(s), and it is unclear whether class I, class II or both sets of peptides are entailed to elicit an optimal immune response [49]. Bacteria present a classy vector for cancer treatment that not only possess the natural ability to grow preferentially with tumors after systemic administration [12] but also, they stimulate innate and flexible immune responses [50] [51]. DNA vaccines stemming from 
bacteria have unmethylated $\mathrm{CpG}$ motifs and stimulate the innate immune responses by interacting with TLR9 expressed on the surface of APCs [52] [53]. This non-specific activation of APCs is likely so as to affect antigen-specific immune responses to DNA vaccines [54].

Today, two methods are commonplace for using bacteria as a vector, and individual genes are inherently suitable for various therapeutic strategies: 1) tumor-specific bacterial reproduction or 2) intracellular plasmid transfer (bactofection). Aggressive bacteria can enter and reproduce with tumor cells, while non-invasive bacteria grow outside of the tumor cells and in the microenvironment of the tumor. The tumor selective growth of bacteria is an attractive mean to deliver reporter genes, therapeutic genes or inhibitory RNA to a tumor for diagnostic and recovery purposes. Now related protocols are presented for the genetic modification of bacterial genomes and the DNA transformation containing genes into bacteria to an increasing range of species. This allows the creation of a bacterium that directly introduces therapeutic genes, and/or efficiently internalize into tumor cells with subsequent therapeutic nucleic acid release for tumor cell presentation ("bactofection"). The same bacterium is engineered to represent a reporter gene(s) that can be used for in vivo imaging of bacterial localization and spread. Bacteria's genetic manipulation can also be expended to create mutations with false or poor nutritional needs, and thus non-pathogens [55].

\section{Conclusion}

In the twenty-first century, the use of bacterial vaccine vectors with an appropriate DNA as a revolution offers the potential options for cancer therapy as a carrier for tumoricidal agents or bacterially directed Enzyme Prodrug therapy. However, it is still a controversial topic that entails more research to be acceptable for clinical and regular treatment for human's tumors. To improve efficacy should work on safety as well as make the tumor environment more hypoxic to attract bacteria [56] [57]. Particular observation should be taken into consideration to prevent lateral gene transfer to other bacteria and to restrict the environmental spread of the vector.

\section{Conflicts of Interest}

The author declares no conflicts of interest regarding the publication of this paper.

\section{References}

[1] Williams, N.S., Bullstrode, C.J. and O’Connell, P.R. (2010) Bailey \& Love's Short Practice of Surgery. Annals of the Royal College of Surgeons of England, 92, 178.

[2] Toussaint, B., Chauchet, X., Wang, Y., Polack, B. and Gouëllec, A. (2013) Live-Attenuated Bacteria as a Cancer Vaccine Vector. Expert Review of Vaccines, 12, 1139-1154. https://doi.org/10.1586/14760584.2013.836914

[3] Egeblad, M., Nakasone, E.S. and Werb, Z. (2010) Tumors as Organs: Complex Tis- 
sues That Interface with the Entire Organism. Developmental Cell, 18, 884-901. https://doi.org/10.1016/j.devcel.2010.05.012

[4] Vaupel, P., Kallinowski, F. and Okunieff, P. (1989) Blood Flow, Oxygen and Nutrient Supply, and Metabolic Microenvironment of Human Tumors: A Review. Cancer Research, 49, 6449-6465.

[5] Elliott, N.T. and Yuan, F. (2011) A Review of Three-Dimensional in Vitro Tissue Models for Drug Discovery and Transport Studies. Journal of Pharmaceutical Sciences, 100, 59-74. https://doi.org/10.1002/jps.22257

[6] Minchinton, A.I. and Tannock, I.F. (2006) Drug Penetration in Solid Tumours. Nature Reviews Cancer, 6, 583-592. https://doi.org/10.1038/nrc1893

[7] Melcher, A., Parato, K., Rooney, C.M. and Bell, J. (2011) Thunder and Lightning: Immunotherapy and Oncolytic Viruses Collide. Molecular Therapy, 19, 1008-1016. https://doi.org/10.1038/mt.2011.65

[8] Lichty, B.D., Breitbach, C.J., Stojdl, D.F. and Bell, J. (2014) Going Viral with Cancer Immunotherapy. Nature Reviews Cancer, 14, 559-567. https://doi.org/10.1038/nrc3770

[9] Roth, J.A. and Cristiano, R. (1997) Gene Therapy for Cancer: What Have We Done and Where Are We Going? Journal of the National Cancer Institute, 89, 21-39. https://doi.org/10.1093/jnci/89.1.21

[10] Kouraklis, G.P. (2003) Gene Therapy for Cancer: Current Status and Prospects. Digestive Diseases and Sciences, 48, 854-855. https://doi.org/10.1023/A:1023026907848

[11] Hall, S.S. and Rosen, F. (1997) A Commotion in the Blood: Life, Death and the Immune System. Nature, 388, 841. https://doi.org/10.1038/42174

[12] Cronin, M., Akin, A.R., Collins, S.A., Meganck, J., Kim, J.-B., Baban, C.K., van Sinderen, D., et al. (2012) High Resolution in Vivo Bioluminescent Imaging for the Study of Bacterial Tumour Targeting. PLoS ONE, 7, e30940. https://doi.org/10.1371/journal.pone.0030940

[13] Zhao, M., Yang, M., Li, X.-M., Jiang, P., Baranov, E., Li, S., Hoffman, R.M., et al. (2005) Tumor-Targeting Bacterial Therapy with Amino Acid Auxotrophs of GFP-Expressing Salmonella typhimurium. Proceedings of the National Academy of Sciences of the United States of America, 102, 755-760. https://doi.org/10.1073/pnas.0408422102

[14] Jaffee, E. (1999) Immunotherapy of Cancer. Annals of the New York Academy of Sciences, 886, 67-72. https://doi.org/10.1111/j.1749-6632.1999.tb09401.x

[15] Dietrich, G., Bubert, A., Gentschev, I., Sokolovic, Z., Simm, A., Catic, A., Goebel, W., et al. (1998) Delivery of Antigen-Encoding Plasmid DNA into the Cytosol of Macrophages by Attenuated Suicide Listeria monocytogenes. Nature Biotechnology, 16, 181-185. https://doi.org/10.1038/nbt0298-181

[16] van Pijkeren, J.P., Morrissey, D., Monk, I.R., Cronin, M., Rajendran, S., O’Sullivan, G.C., Tangney, M., et al. (2010) A Novel Listeria monocytogenes-Based DNA Delivery System for Cancer Gene Therapy. Human Gene Therapy, 21, 405-416. https://doi.org/10.1089/hum.2009.022

[17] Medina, E. and Guzmán, C. (2001) Use of Live Bacterial Vaccine Vectors for Antigen Delivery: Potential and Limitations. Vaccine, 19, 1573-1580. https://doi.org/10.1016/S0264-410X(00)00354-6

[18] Garmory, H.S., Leary, S.E., Griffin, K.F., Diane Williamson, E., Brown, K.A. and Titball, R. (2003) The Use of Live Attenuated Bacteria as a Delivery System for He- 
terologous Antigens. Journal of Drug Targeting, 11, 471-479. https://doi.org/10.1080/10611860410001670008

[19] Jiang, S.-N., Phan, T.X., Nam, T.-K., Nguyen, V.H., Kim, H.-S., Bom, H.-S., Min, J.-J., et al. (2010) Inhibition of Tumor Growth and Metastasis by a Combination of Escherichia coli-Mediated Cytolytic Therapy and Radiotherapy. Molecular Therapy, 18, 635-642. https://doi.org/10.1038/mt.2009.295

[20] Weibel, S., Stritzker, J., Eck, M., Goebel, W. and Szalay, A. (2008) Colonization of Experimental Murine Breast Tumours by Escherichia coli K-12 Significantly Alters the Tumour Microenvironment. Cellular Microbiology, 10, 1235-1248. https://doi.org/10.1111/j.1462-5822.2008.01122.x

[21] Carleton, H. (2010) Combating Evolving Pathogens Pathogenic Bacteria as Vaccine Vectors: Teaching Old Bugs New Tricks. Yale Journal of Biology and Medicine, 83, 217-222.

[22] Toussaint, B., Chauchet, X., Wang, Y., Polack, B. and Gouëllec, A. (2013) Live-Attenuated Bacteria as a Cancer Vaccine Vector. Expert Review of Vaccines, 12, 1139-1154. https://doi.org/10.1586/14760584.2013.836914

[23] Radvanyi, L. (2004) Discovery and Immunologic Validation of New Antigens for Therapeutic Cancer Vaccines. International Archives of Allergy and Immunology, 133, 179-197. https://doi.org/10.1159/000076625

[24] Bolhassani, A. and Zahedifard, F. (2012) Therapeutic Live Vaccines as a Potential Anticancer Strategy. International Journal of Cancer, 131, 1733-1743.

https://doi.org/10.1002/ijc. 27640

[25] Attridge, S.R., Davies, R. and LaBrooy, J. (1997) Oral Delivery of Foreign Antigens by Attenuated Salmonella: Consequences of Prior Exposure to the Vector Strain. Vaccine, 15, 155-162. https://doi.org/10.1016/S0264-410X(96)00158-2

[26] Coley, W. (1894) Treatment of Inoperable Malignant Tumors with the Toxines of Erysipelas and the Bacillus Prodigiosus. The American Journal of the Medical Sciences, 108, 50-66. https://doi.org/10.1097/00000441-189407000-00006

[27] Tsung, K. and Norton, J.A. (2006) Lessons from Coley's Toxin. Surgical Oncology, 15, 25-28. https://doi.org/10.1016/j.suronc.2006.05.002

[28] Patyar, S., Prakash, A. and Medhi, B. (2012) Bacteria as a Therapeutic Approach in Cancer Therapy. In: Bacteria and Cancer, Springer, Berlin, 185-208. https://doi.org/10.1007/978-94-007-2585-0_8

[29] Saltzman, D.A., Heise, C.P., Hasz, D.E., Zebede, M., Kelly, S.M. and Curtiss III, R. (1996) Attenuated Salmonella Typhimurium Containing Interleukin-2 Decreases MC-38 Hepatic Metastases: A Novel Anti-Tumor Agent. Cancer Biotherapy and Radiopharmaceuticals, 11, 145-153. https://doi.org/10.1089/cbr.1996.11.145

[30] Saltzman, D.A., Katsanis, E., Heise, C.P., Hasz, D.E., Kelly, S.M. and Curtiss III, R. (1997) Patterns of Hepatic and Splenic Colonization by an Attenuated Strain of Salmonella Typhimurium Containing the Gene for Human Interleukin-2: A Novel Anti-Tumor Agent. Cancer Biotherapy and Radiopharmaceuticals, 12, 37-45. https://doi.org/10.1089/cbr.1997.12.37

[31] Yuhua, L., Kunyuan, G., Hui, C., Yongmei, X., Chaoyang, S., Xun, T. and Daming, R. (2001) Oral Cytokine Gene Therapy against Murine Tumor Using Attenuated Salmonella Typhimurium. International Journal of Cancer, 94, 438-443. https://doi.org/10.1002/ijc.1489

[32] Li, X., Fu, G.-F., Fan, Y.-R., Liu, W.-H., Liu, X.-J., Wang, J.-J. and Xu, G.-X. (2003) Bifidobacterium adolescentis as a Delivery System of Endostatin for Cancer Gene Therapy: Selective Inhibitor of Angiogenesis and Hypoxic Tumor Growth. Cancer 
Gene Therapy, 10, 105-111. https://doi.org/10.1038/sj.cgt.7700530

[33] Yasui, H. and Ohwaki, M. (1991) Enhancement of Immune Response in Peyer's Patch Cells Cultured with Bifidobacterium Breve. Journal of Dairy Science, 74, 1187-1195. https://doi.org/10.3168/jds.S0022-0302(91)78272-6

[34] Reddy, B.S. and Rivenson, A. (1993) Inhibitory Effect of Bifidobacterium Longum on Colon, Mammary, and Liver Carcinogenesis Induced by 2-Amino-3-methylimidazo[4][5-f]quinoline, a Food Mutagen. Cancer Research, 53, 3914-3918.

[35] Brüggemann, H. and Gottschalk, G. (2009) Clostridia: Molecular Biology in the Post-Genomic Era. Caister Academic Press. http://hdl.handle.net/11858/00-001M-0000-000E-C121-0

[36] Theys, J., Landuyt, W., Nuyts, S., Van Mellaert, L., Van Oosterom, A., Lambin, P. and Anné, J. (2001) Specific Targeting of Cytosine Deaminase to Solid Tumors by Engineered Clostridium acetobutylicum. Cancer Gene Therapy, 8, 294-297. https://doi.org/10.1038/sj.cgt.7700303

[37] Liu, S., Minton, N., Giaccia, A. and Brown, J. (2002) Anticancer Efficacy of Systemically Delivered Anaerobic Bacteria as Gene Therapy Vectors Targeting Tumor Hypoxia/Necrosis. Gene Therapy, 9, 291-296. https://doi.org/10.1038/sj.gt.3301659

[38] Luo, X., Li, Z., Shen, S., Runyan, J., Bermudes, D. and Zheng, L. (2001) Genetically Armed Salmonella Typhimurium Delivered Therapeutic Gene and Inhibited Tumor Growth in Preclinical Models. Proceedings of Annual Meeting at American Association for Cancer Research, 42, 3693.

[39] Pawelek, J.M., Low, K.B. and Bermudes, D. (1997) Tumor-Targeted Salmonella as a Novel Anticancer Vector. Cancer Research, 57, 4537-4544.

[40] Fujimori, M., Amano, J. and Taniguchi, S. (2002) The Genus Bifidobacterium for Cancer Gene Therapy. Current Opinion in Drug Discovery \& Development, 5 , 200-203.

[41] Cheng, C., Lu, Y., Chuang, K., Hung, W., Shiea, J., Su, Y., Cheng, T., et al. (2008) Tumor-Targeting Prodrug-Activating Bacteria for Cancer Therapy. Cancer Gene Therapy, 15, 393-401. https://doi.org/10.1038/cgt.2008.10

[42] Cheever, M.A., Allison, J.P., Ferris, A.S., Finn, O.J., Hastings, B.M., Hecht, T.T. and Weiner, L. (2009) The Prioritization of Cancer Antigens: A National Cancer Institute Pilot Project for the Acceleration of Translational Research. Clinical Cancer Research, 15, 5323-5337. https://doi.org/10.1158/1078-0432.CCR-09-0737

[43] Kufe, D.W., Hait, W., Holland, J.F., Frei, E. and Pollock, R.E. (2010) Holland-Frei Cancer Medicine 8 (Vol. 8). PMPH-USA.

[44] Wood, L.M. and Paterson, Y. (2014) Attenuated Listeria monocytogenes. A Powerful and Versatile Vector for the Future of Tumor Immunotherapy. Frontiers in Cellular and Infection Microbiology, 4, 51. https://doi.org/10.3389/fcimb.2014.00051

[45] Dalpke, A., Zimmermann, S. and Heeg, K. (2001) CpG-Oligonucleotides in Vaccination: Signaling and Mechanisms of Action. Immunobiology, 204, 667-676. https://doi.org/10.1078/0171-2985-00106

[46] Krieg, A.M., Yi, A.-K., Matson, S., Waldschmidt, T.J., Bishop, G.A., Teasdale, R., Klinman, D., et al. (1995) CpG Motifs in Bacterial DNA Trigger Direct B-Cell Activation. Nature, 374, 546. https://doi.org/10.1038/374546a0

[47] Yanai, H., Ban, T. and Taniguchi, T. (2011) Essential Role of High-Mobility Group Box Proteins in Nucleic Acid-Mediated Innate Immune Responses. Journal of Internal Medicine, 270, 301-308. https://doi.org/10.1111/j.1365-2796.2011.02433.x 
[48] Schlom, J., Hodge, J.W., Palena, C., Tsang, K.-Y., Jochems, C., Greiner, J.W., Gulley, J.L., et al. (2014) Therapeutic Cancer Vaccines. In: Advances in Cancer Research, Vol. 121, Elsevier, Amsterdam, 67-124. https://doi.org/10.1016/B978-0-12-800249-0.00002-0

[49] Kaufman, H.L. (2012) Vaccines for Melanoma and Renal Cell Carcinoma. Seminars in Oncology, 39, 263-275. https://doi.org/10.1053/j.seminoncol.2012.02.011

[50] Singh, R. and Paterson, Y. (2006) Listeria monocytogenes as a Vector for Tumor-Associated Antigens for Cancer Immunotherapy. Expert Review of Vaccines, 5, 541-552. https://doi.org/10.1586/14760584.5.4.541

[51] Haux, J. (2001) Infection and Cancer. The Lancet, 358, 155-156. https://doi.org/10.1016/S0140-6736(01)05369-7

[52] Liu, M.A. and Ulmer, J. (2005) Human Clinical Trials of Plasmid DNA Vaccines. Advances in Genetics, 55, 25-40. https://doi.org/10.1016/S0065-2660(05)55002-8

[53] Sato, Y., Roman, M., Tighe, H., Lee, D., Corr, M., Nguyen, M.-D., Raz, E., et al. (1996) Immunostimulatory DNA Sequences Necessary for Effective Intradermal Gene Immunization. Science, 273, 352-354. https://doi.org/10.1126/science.273.5273.352

[54] Lee, S.-H., Danishmalik, S.N., Sin, J.-I., et al. (2015) DNA Vaccines, Electroporation and Their Applications in Cancer Treatment. Human Vaccines \& Immunotherapeutics, 11, 1889-1900. https://doi.org/10.1080/21645515.2015.1035502

[55] Cronin, M., Stanton, R., Francis, K. and Tangney, M. (2012) Bacterial Vectors for Imaging and Cancer Gene Therapy: A Review. Cancer Gene Therapy, 19, 731-740. https://doi.org/10.1038/cgt.2012.59

[56] Dietzel, F. (1983) Basic Principles in Hyperthermic Tumor Therapy. In: Vascular Perfusion in Cancer Therapy, Springer, Berlin, 177-190. https://doi.org/10.1007/978-3-642-82025-0_31

[57] Dietzel, F. and Gericke, D. (1977) Intensification of the Oncolysis by Clostridia by Means of Radio-Frequency Hyperthermy in Experiments on Animals-Dependence on Dosage and on Intervals (Author's Transl.) Strahlentherapie, 153, 263-266. 